\title{
High Level Information Fusion Developments, Issues, and Grand Challenges Fusion2010 Panel Discussion
}

\author{
Erik Blasch \\ Defence R\&D Canada-Valcartier \\ Québec City, QC, Canada \\ erik.blasch@drdc-rddc.gc.ca
}

\author{
Subrata Das \\ Xerox \\ Grenoble, France \\ subrata.das@xrce.xerox.com
}

\author{
James Llinas \\ SUNY - Buffalo \\ Buffalo, NY, USA \\ llinas@buffalo.edu \\ Chee Chong \\ BAE Systems \\ Los Altos, CA, USA \\ chee.chong@baesystems.com
}

\author{
Dale Lambert \\ Defence Science \& Tech. Org. \\ Edinburgh, SA, Australia \\ dale.lambert@dsto.defence.gov.au \\ Mitch Kokar \\ Northeastern University \\ Cambridge, MA, USA \\ kokar@coe.neu.edu
}

\author{
Pierre Valin \\ Defence R\&D Canada-Valcartier \\ Québec City, QC, Canada \\ pierre.valin@drdc-rddc.gc.ca
}

\author{
Elisa Shahbazian \\ OODA Technologies \\ Montréal, QC, Canada \\ elisa.shahbazian@ooda.ca
}

\begin{abstract}
The goal of the High-Level Information Fusion (HLIF) Panel Discussion is to present contemporary HLIF advances and developments to determine unsolved grand challenges and issues. The discussion will address the issues between low-level (signal processing and object state estimation and characterization) and high-level information fusion (control, situational understanding, and relationships to the environment). Specific areas of interest include modeling (situations, environments), representations (semantic, knowledge, and complex), systems design (scenario-based, user-based, distributedagent) and evaluation (measures of effectiveness and empirical case studies). The goal is to address the contemporary operational and strategic issues in information fusion system design.
\end{abstract}

Keywords: Fusion, Situational/Impact Assessment, Resource/Sensor Management, User Refinement

\section{Panel Motivation}

High-level Information Fusion (HLIF) has been of considerable interest to the fusion community ever since the development of the fusion process models. The lowlevel versus high-level distinction was made evident in the seminal text on the subject by Waltz and Llinas, Multisensor Data Fusion, in "Figure 1.1 Elements of a basic data fusion system.” [1] While many discussions in HLIF have been coordinated in the past decade at the fusion conferences, including other panel discussions, there is a need to gather contemporary insights into the ongoing challenges. Recent HLIF texts include: Mathematical Techniques in Multisensor Data Fusion [2], Concepts, Models, and Tools for Information Fusion [3], High-Level Fusion [4], and Handbook of Multisensor Data Fusion, [5-6].

\subsection{Panel Organization and Discussion Overview}

For this panel, experts were compiled based on various research thrusts:

Modeling: Lambert, Das, Kokar

Representation: Blasch, Valin, Kokar
Systems Design: Llinas, Das, Chong

Decision Support: Valin, Llinas, Shahbazian

Evaluation Methods: Blasch, Llinas, Valin

The HLIF panel discussion's goal is to highlight the unsolved problems and concerns to motivate the information fusion community towards systems-level solutions. The panelists' expert perspectives are based on three areas: (1) previous panel discussions and summaries, (2) an integrated list of HLIF challenges, and (3) companion papers presented at the Fusion2010 conference (note we switch to Fusion10 to refer to the conference).

\subsection{Previous Related Panel Discussions}

Panel discussions provide a valuable resource to the community to overview the current techniques and provide areas of concern for future research. Previous Fusion Conference panel discussion papers related to HLIF include knowledge representation (Fusion05) [7], resource management coordination with situation and threat assessment (Fusion06) $[\mathbf{8}, \mathbf{9}, \mathbf{1 0}]$, agent-based design (Fusion07) [11], and HLIF challenges (Fusion08) [12]. Three panel discussions were conducted at Fusion09 without papers:

I. Kadar, M. Sudit, A. Steinberg, J. Roy, G. Toth, and J. Salerno, Issues and Challenges in Higher-Level Fusion Threat and Impact Assessment.

G. Toth, M. Kokar, M. Liggins, G. Powell, J. Salerno, M. Endsley, A. Pfeffer, C. Knoblock, Directions for HigherLevel Fusion Research, Needs and Capabilities.

D. Lambert, M. Hinman, E. Bossé, and C. Blackman, A Coalition Approach to Higher-Level Fusion.

Many of the authors of this Fusion10 HLIF panel coordinated on previous publications, but continual refinement of HLIF contemporary are desired. The panel discussion follows from a day-long event special session. There are most likely other papers at Fusion10 that are related that would validate good questions from the audience to the panelists. Many of the participants to the special session would be encouraged to voice their opinions and questions to the moderated panel. 


\subsection{HLIF Grand Challenges}

It is at the last Fusion09 panel discussion Coalition Approach to Higher-Level Fusion that this group was organized. From the discussion, five areas of interest that pose grand challenges for the information fusion community include:

(1) How to model (e.g. formal theories) and control a situation using a systems-level fusion context?

(2) What constitutes situational, semantic, belief and knowledge representations?

(3) How to design distributed systems and incorporate scenario-based design approaches?

(4) When and where are user-system interactions coordinated in the fusion system`s decision support? , and

(5) What are the metrics and visualizations needed for effective evaluation of HLIF systems?

Note that the discussion of higher-level fusion architectures have not specifically addressed who are the users for the various systems; whether it be an operator, commander, or design engineer.

For the Fusion10 panel discussion participants, questions of interest from the five areas include:

What techniques and procedures are most applicable?

What are the tacit implications for HLIF?

What is needed in HLIF to support control?

What is the impact of HLIF to decision support?

What constitutes effective system evaluation?

Answers to these questions were formulated in the companion Fusion10 papers.

\subsection{Fusion10 Panel Papers Overview}

The Fusion10 papers in support of the panelist's current directions are as follows:

Formal theories for HLIF SA modeling $[13,14,15]$. Situation and Knowledge Representations [16, 17]

HLIF system design $[\mathbf{1 8}, \mathbf{1 9}]$

HLIF for decision support [20]

HLIF evaluation [21, 22]

Three common themes throughout the papers include:

(A) Information fusion designs support situational awareness. Advanced techniques in design (e.g. agent-based) and formal theories are needed to support contextual understanding and information management. Common prototypes and testbeds are needed for comparative evaluation of techniques.

(B) The fusion process has a requirement for a layered set of adaptive process control loops of various types (i.e. between fusion processes and within a level, inter-level control, and sensor/information management). Distributed control issues are a critical key element of design and implementation of any fusion process yet receives little attention in the community.

(C) Understanding feasible solutions and the role of human intelligence. Today, we are facing complex, dynamic problem environments and new input modalities (text/language) that impute entirely new challenges. We need to understand what aspects of these problems can be addressed with automated machine-processing methods and where and to what extent we need human intelligence inserted. There is little to no calibration of what levels of complexity and dimensionality a HLIF system can support users via automated operations. A successful HLIF system should combine machine computing power with human cognition/intuition.

The rest of this paper includes an overview of highinformation level fusion in Section 2. Section 3 provides previous statements of information fusion challenges and Section 4 review the literature from past fusion conferences on HLIF. The literature review provides a basis that motivates the panel discussion from which conclusions are drawn in Section 5.

\section{Introduction to HLIF}

The distinction between high-level fusion (HLIF) and low-level fusion (LLIF) was first made evident by Waltz and Llinas in the classical text in information fusion (shown in Figure 1) [1]. The low-level functional processes support target classification, identification, and tracking, while high-level functional processes support situation, impact, and fusion process refinement. LLIF concerns numerical data (e.g., locations, kinematics, and attribute target types). HLIF concerns abstract symbolic information (e.g., threat, intent, and goals).

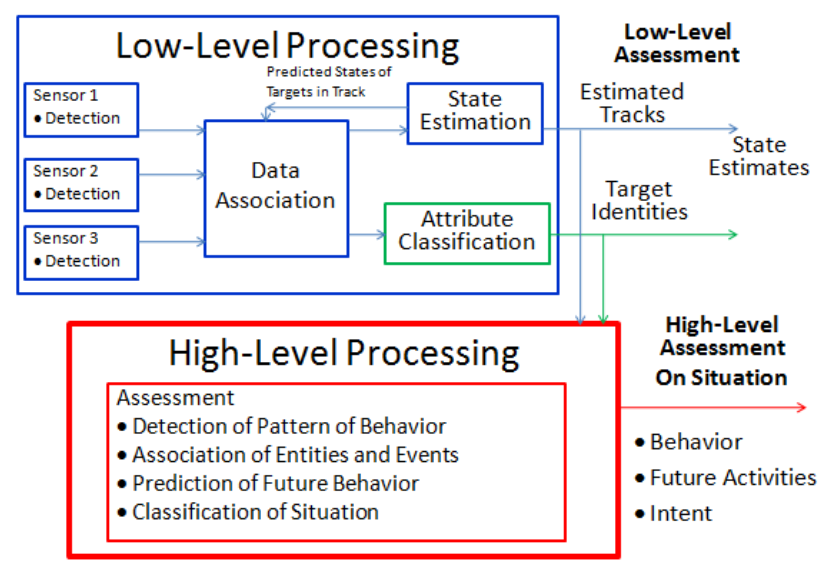

Figure 1 Elements of a basic data fusion system. Adapted from E. Waltz and J. Llinas, Multisensor Data Fusion, Artech House, Norwood, MA [1990]) 
Following [1], the Joint Directors of Laboratories (JDL) model was proposed [23]. Subsequent revisions [24, 25] were made to the model to incorporate new understandings of the issues involved in developing an information fusion system. In 2004, the JDL model was revised by the Data Fusion Information Group (DFIG).

\subsection{DFIG Fusion Model}

The DFIG model [7] supports the original JDL goals while highlighting pragmatic design issues by coupling various resource management (RM) functions with information fusion (IF) estimation needs. The DFIG model $^{1}$ supports differing control functions based on the spatial/temporal/ spectrum differences. The spectral needs drive sensor selection. The temporal needs are based on the user's need for timely information to afford action. Finally, the spatial needs are based on the mission goals. The current team diagrammed the current process model, shown in Figure 2, while maintaining the structure of the JDL model.

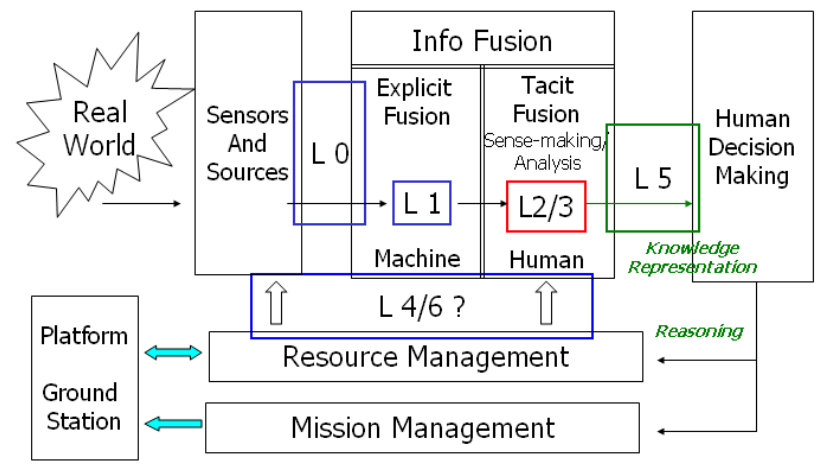

Figure 2. Data Fusion Information Group (DFIG) model.

The current DFIG definitions include:

Level 0 - Data Assessment: estimation and prediction of signal/object observable states on the basis of pixel/signal level data association (e.g. information systems collections);

Level 1 - Object Assessment: estimation and prediction of entity states on the basis of data association, continuous state estimation and discrete state estimation (e.g. data processing);

Level 2 - Situation Assessment: estimation and prediction of relations among entities, to include force structure and force relations, communications, etc. (e.g. information processing);

Level 3 - Impact Assessment: estimation and prediction of effects on situations of planned or estimated actions by the participants; to include interactions between action plans of multiple players (e.g. assessing threat /intent actions to planned actions and mission requirements, performance evaluation);

Level 4 - Process Refinement (an element of Resource Management): adaptive data acquisition and processing to support sensing objectives (e.g. fusion process control and information systems dissemination).

Level 5 - User Refinement (an element of Knowledge Management): adaptive determination of who queries information and who has access to information (e.g. information operations) and adaptive data retrieved and displayed to support cognitive decision making and actions (e.g. human computer interface).

\footnotetext{
${ }^{1}$ Frank White, Otto Kessler, Chris Bowman, James Llinas, Erik Blasch, Gerald Powell, Mike Hinman, Ed Waltz, Dale Walsh, John Salerno, Alan Steinberg, Dave Hall, Ron Mahler, Mitch Kokar, Joe Karalowski, Richard Antony
}

Level 6 - Mission Management (an element of Platform Management): adaptive determination of spatial-temporal control of assets (e.g. airspace operations) and route planning and goal determination to support team decision making and actions (e.g. theater operations) over social, economic, and political constraints.

In the DFIG model, the goal was to separate the IF and $\mathrm{RM}$ functions. RM is divided into sensor control, platform placement, and user selection to meet mission objectives. L2 (SA) includes tacit functions which are inferred from L1 explicit representations of object assessment. Since the unobserved aspects of the SA problem cannot be processed by a computer, user knowledge and reasoning is necessary. L3 (IA) sense-making of impacts (threats, course of actions, game-theoretic decisions, intent, etc.) helps refine the SA estimation and information needs for different actions.

High-level information fusion (as referenced to levels beyond the DFIG Model Level 1) is the ability of a fusion system, through knowledge, expertise, and understanding to: capture awareness and complex relations, reason over past and future events, utilize direct sensing exploitations and tacit reports, and discern the usefulness and intention of results to meet system-level goals. The Information Fusion community has coined the term "high-level fusion" however this implies that there is a low-level / high-level distinction when in reality they are coupled. Designs of real-world Information Fusion Systems imply distributed information source coordination (network), organizational concepts (command), and environmental understanding (context). There is a need for automated processes that provide functionality in support of user decision processes, particularly at higher levels requiring reasoning and inference.

\subsection{State Transition Data Fusion Model}

As functional models, variants of the JDL model celebrate the differences between the sub-object, object, situation and impact assessment JDL levels at the expense of highlighting their commonality. In 2006, Lambert [26] responded by introducing the STDF model, which rests upon three unifying tenets aimed at exposing the essence of data fusion.

(1) Situation awareness is fusion performed by people, while machine fusion is "situation awareness" performed by machines. If "sensation" is added as a level 0 to Endley's [27] definition of situation awareness, then there is a direct correspondence between levels 0 to 3 of situation awareness and levels 0 to 3 of machine fusion respectively [28, 29]. The adaptive level 4 can be partitioned across levels 0 to 3 . Level 5 then comprises levels 0 to 3 being performed by a human. Fusion at levels 0 to 3 can then be understood as being performed by people, machines, or some combination of the two. The appropriate level of automation for each of these fusion levels should be decided empirically [30]. Some aspects are better 
handled by people, while others are better performed by machines.

(2) At each of the JDL levels 0 to 3, the world can be understood in terms of transitions between states. Conceptualizing the world as transitions between states is a common theme for each of the JDL levels 0 to 3 . What differs at each level is the notion of state, which acquires increasing numerical to symbolic complexity across the levels. Figure 3 illustrates the nature of state transitions across levels 0 to 3 and the corresponding human and machine fusion processes associated with them.

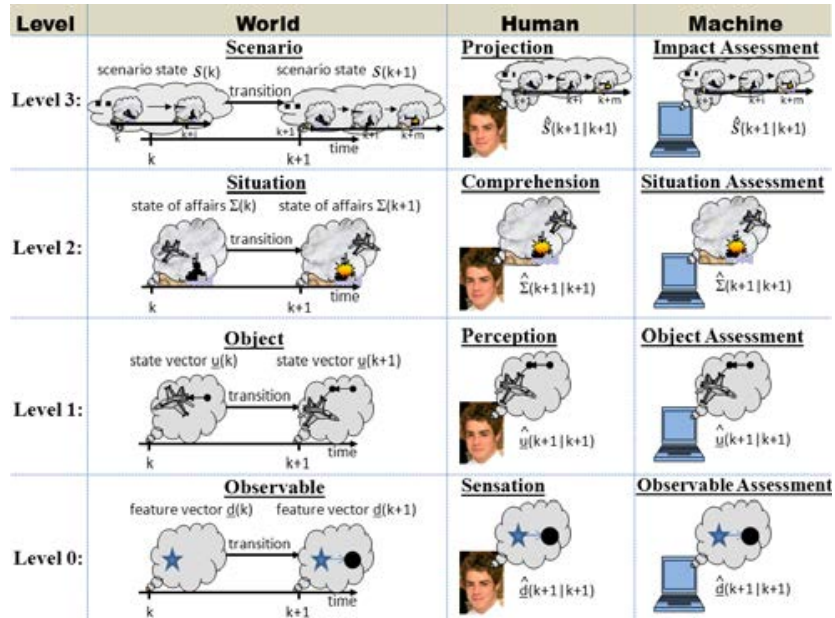

Figure 3. STDF State Transitions At Each Level

(3) At each of the JDL levels 0 to 3, a common fusion process applies that aims to explain the world through prediction and observation. The generic fusion process predicts to observe, observes to explain, and explains to predict. What differs at each level is how the component processes are realized, given the nature of states at that level. Some component processes represent the STDF fusion process operating at a different level. When interpreted at level 2, for example, the STDF model "detection" process is in fact the whole object assessment STDF process of level 1.

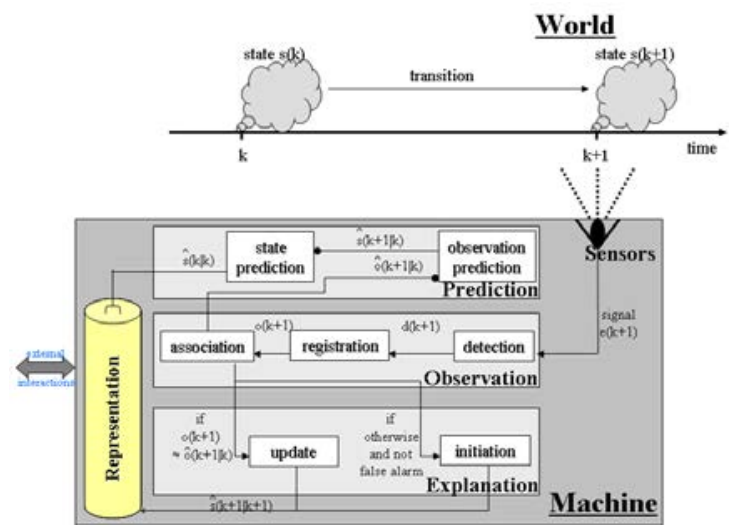

Figure 4. Generic STDF Fusion Process.
In [28] Lambert presents a general uncertainty framework for the STDF model while [31] identifies a blueprint for its implementation.

While the various information fusion models and architectures support conceptualizations of fusion process; there are many daunting challenges for delivering an operational system. There have been many guidelines of information fusion challenges that motivate the community for active research.

\section{Information Fusion Challenges}

Early work by Hall and Llinas in 1997 [32] addressed various challenges for information fusion as aligned with the information fusion level notation. For example in Level 4, they list the key techniques of Measurement of Effectiveness (MOE), Measures of performance (MOP), and Utility theory as well as attention to mission management with issues and challenges listed below:

\begin{tabular}{|c|c|}
\hline Current Status & Challenges and Limitations \\
\hline $\begin{array}{l}\text { Robust system for single- } \\
\text { sensor sys. }\end{array}$ & $\begin{array}{l}\text { Incorporation of mission } \\
\text { objectives/constraints }\end{array}$ \\
\hline Operations research & Environmental context for sensor utilization \\
\hline formulation & Conflicting objectives (e.g. detection vs. \\
\hline Limited approximate & accuracy) \\
\hline reasoning app. & Dynamic algorithm selection/modification \\
\hline Focus on MOP and MOE & Diverse sensors \\
\hline
\end{tabular}

The key challenges expressed were (1) limited communications bandwidth for data aggregation, (2) context-based approximate reasoning for L3 understanding, and (3) knowledge representation for L2 processing, which were similar issues of the Fusion05 panel discussion for SA processing [7]. The interplay between RM and the various high-level processes are still evolving as more data becomes available and information fusion techniques are applied to large contextual applications.

\subsection{Lambert's Grand Challenges}

Dale Lambert [33] posed some grand challenges for the Information community in 2003 to include:

Semantic Challenge: What symbols should be used and how do those symbols acquire meaning?

Epistemic Challenge: What information should we represent and how should it be represented and processed within the machine?

Paradigm Challenge: How should the interdependency between the sensor fusion and information fusion paradigms be managed?

Interface Challenge: How do we interface people to complex symbolic information stored within machines?

System Challenge: How should we manage data fusion systems formed from combinations of people and machines? 
The grand challenges relate to the need to incorporate the human in the decision process (i.e. Level 5, "User Refinement” [2, 6, 34]). Likewise, there were representation, design, and decision support challenges. The implied modeling challenges pose the need for syntactic, semantic, and pragmatic solutions. What is added to the original grand challenge list are evaluation challenges.

\subsection{Previous Fusion Panel Discussion Challenges}

\subsubsection{Situation Assessment (Level 2)}

From the invited Fusion05 panel discussion, "Issues and Challenges of Knowledge Representation and Reasoning Methods in Situation Assessment (Level 2) fusion”, the common themes and challenges were [7]:

\section{ISSUES}

User - The SA process includes perceptual, interactive, and human control

Process models - updating behavioral models (e.g. - Bayes Nets, procedural/logical, perceptual, learning)

Context - operational situation (i.e. dependent on the current state of the environment)

Meaning - semantics and syntax issues (formal methods, ontologies)

Metrics - develop a standard set of metrics (e.g. trust, bounds, uncertainty)

\section{CHALLENGES}

Explanation of the process - evidence accumulation and contradiction detection in reasoning

Graphical displays to facilitate inferential chains, collaborative interaction, and knowledge representation

Interactive control for corrections and utility assessment for knowledge management

\subsubsection{Resource Management (Level 4)}

An invited 2006 panel discussion, entitled "Issues and Challenges in Resource Management with Applications to Real-World Problems" addressed these challenges: [8]

(1) formulating utility functions,

(2) distributed attention,

(3) net-centric network and service management bandwidth allocation for $\mathrm{L} 1 / 2 / 3$,

(4) distributed algorithms with adaptive platforms and sensors,

(5) off-line learning combined with real-time optimization, and

(6) performance metrics.

From the Fusion06 panel discussion, "Resource Management and its Interactions with Level 2/3 Fusion" [9], the common themes were:

(1) Addressing the user in system management / control,

(2) Determining a standard set of metrics for optimization,

(3) Optimizing / evaluating fusion systems to deliver timely information needs,

(4) Dynamic updating for planning mission time-horizons,

(5) Joint optimization of objective functions at all levels
(6) L2/3 situation entity definitions for knowledge discovery, modeling, and information projection

(7) Addressing constraints for resource planning and scheduling

The RM tradeoffs, design attributes, and challenges for instantiating this model include:

Issues for level 2/3 analysis with L4 control include:

1) Level $2 / 3$ tradeoffs in information quantity (throughput)

2) Timeliness of process refinement for sensing control, information updating, and mission planning needs

3) Level 3 domain knowledge context use to predict future needs

4) Multiple distributed users have differing levels of processing needs for the same situation

5) Varying fidelity of confidence reporting of impending threats and situations based on uncertainty calculus

The IF challenges include the development of:

1) Pedigree analysis to backtrack through associations to capture the impending threat

2) Time Horizons of control actions from IA to update the SA (i.e. priority schemes)

3) Performance models of L1 analysis to afford L2/3 information needs satisfaction and level 4 RM.

4) Hierarchical cost functions that include risk and utility analysis of L4 processes.

5) Unified set of metrics that afford SA/IA processing that can be jointly optimized in a RM 4 objective function

6) Communication and timely ordering of information to include constraints for resource planning and scheduling and network service management, and

7) L2/3 situation entity definitions for knowledge discovery, modeling, and information projection.

\subsubsection{High-Level Fusion}

From the Fusion08 Panel Discussion, "Higher-Level Information Fusion Challenges to the Academic Community,” [11] these challenges were addressed:

(1) Appropriate process models for the fusion process

(2) "Estimation"-like capabilities for HLIF relations

(3) Formal models for HLIF

(4) Use of other domains such as computer science to foster Information Fusion solutions.

As discussed in Section 1.3, the goal of the Fusion10 HLIF Panel Discussion is bring together advances and developments in HLIF with interest in determining the contemporary challenges. The discussion focuses on the issues between low-level (signal processing, object state estimation and characterization) and high-level fusion (control and relationships to the environment). Specific areas of interest include modeling (situations, environments), representations (semantic, knowledge, and complex), systems design (scenario-based, user-based, distributed-agent) and evaluation (measures of performance/effectiveness, and empirical case studies). The goal is to address the operational and strategic issues in pragmatic information Fusion system designs. 


\section{HLIF Over the Last Decade}

This section serves as a retrospective view of key issues and challenges addressed from the fusion community. The reader is referred to the individual papers [www.isif.org] for specific documentations. One note is that the papers referenced addressed "higher-level fusion," although other notions of specific issues of higher-level information fusion (L2-L5) could have been addressed in other papers.

\subsection{From SA/TA to User Refinement}

From 2001 to 2002, researchers were mostly concerned with situation assessment [35, 36, 37, 38]. Sycara and Lewis $[39,40]$ focus on user issues including decision making, semantics, and actionable information.

Fusion03 incorporates differing HLIF issues and solutions to situation assessment [41, 42, 43] and intent estimation [44]. A new theme emerges in ontology representations [45, 46, 47].

Fusion04 HLIF research includes situational presentations $[48,49,50]$ of context dependent attributes.

In 2005, Schubert and Svensson provide a first of a kind literature review of robust high level fusion performance [51]. Also, in the Fusion05 conference, Lambert [52] expands upon his semantic challenges. Likewise Kokar [53, 54] addresses HLIF situation awareness solutions. Salerno [55] addresses SA evaluation issues. Process refinement [56, 57] and user refinement [58] are presented.

Fusion06 includes HLIF fusion theories for SA from Lambert [59, 60], threat assessment HLIF issues [61], and the importance of decision support $[62,63]$. Only two papers in Fusion07 specifically discuss HLIF designs [64, 65]. In 2008, the HLIF papers mainly focus on threat assessment evaluation and assessment $[\mathbf{6 6}, 6 \mathbf{6 7}, 68]$.

\subsection{HLIF as an Emerging Topic}

During 2009, with the already mentioned numerous panels calling out the needs for HLIF, numerous papers are presented. Solutions are presented for HLIF L2 situation assessment [69, 70, 71] and L3 threat assessment [72]. The scenario issues of context $[\mathbf{7 3}, \mathbf{7 4}, \mathbf{7 5}]$ and culture [76] are addressed. Various L5 user refinement decision support techniques are proposed [77, 78, 79]. Finally system design issues are presented with metrics [80] and evaluation [81].

\subsection{Discussion on High-level Fusion}

The term "high-level fusion" is contrasted to "low-level fusion" in numerous papers that include image processing, ontology, and robotics. In many cases, the authors dictate a distinction of their own algorithms from data and information aggregation. These papers do not refer to the Information Fusion community levels, but delineate the discussion in their own architectures. For example, in robotics, [82], low-level fusion is defined as direct integration of sensory data, resulting in parameter and state estimates; whereas high-level fusion is used for indirect integration of sensory data through command arbitration of control signals suggested by different hierarchical modules. Many robotics and image processing papers make distinctions between estimation and control, which was the basis of the JDL modifications [23-25], however they focus on data versus information fusion.

There are many ideas the fusion community can leverage in support of HLIF designs. Conferences on belief reasoning (COGSCI), situation assessment and management (SIMA), and contextual understanding (MORS); can provide insights into HLIF designs.

\section{Summary of Panel Discussion}

High-level fusion (Situation and Threat Assessment, Process and User Refinement) requires novel solutions for the transition of information fusion designs. There are numerous ongoing challenges that the Fusion community can discuss towards a common understanding and coordination. Current panel thoughts have highlighted these five grand challenges for HLIF:

1) HLIF Modeling (situations, environments),

2) Representations of HLIF Information (semantic, knowledge, and complex),

3) Systems design techniques (scenario-based, userbased, and distributed-agent)

4) Decision support processes (reasoning, inference, and relationships), and

5) Evaluation methods (measures of performance/ effectiveness, and empirical case studies).

\section{References}

[1] E. Waltz and J. Llinas, Multisensor Data Fusion, Artech House, Norwood, MA, 1990.

[2] D. L. Hall and S. A. McMullen, Mathematical Techniques in Multisensor Data Fusion, Artech, 2004.

[3] E. Bossé, J. Roy, and S. Wark, Concepts, Models, and Tools for Information Fusion, Artech House, 2007.

[4] S. Das, High-Level Data Fusion, Artech House, 2008.

[5] J. Llinas, "Assessing the Performance of Multisensor Fusion Processes," Ch 20 in Handbook of Multisensor Data Fusion, (Eds.) D. Hall and J. Llinas, CRC Press, 2001.

[6] E. Blasch, "User refinement in Information Fusion", Chapter 19 in Handbook of Multisensor Data Fusion $2^{\text {nd }}$ Ed, (Eds.) D. Hall, and J. Llinas, CRC Press, 2008.

PANELS

[7] E. Blasch, I. Kadar, J. Salerno, M. Kokar, S. Das, G. Powell, D. Corkill, and E. Ruspini, "Issues and Challenges of Knowledge Representation and Reasoning Methods in Situation Assessment (Level 2) fusion”, Journal for Advances in Information Fusion, 2006.

[8] I. Kadar, R. Mahler, T. Kirubarajan, D. Castanon, M. Farooq, K. Hintz, J. Reich, "Panel Discussion: Issues in Resource Management with Applications to Real-World Problems," Signal Proc., Sensor Fusion, and ATR, XV, Vol. 6235, SPIE, 2006. 
[9] E. Blasch, I. Kadar, K. Hintz, J. Biermann, C. Chong, J. Salerno, and S. Das, "Resource Management and Its Interactions with Level 2/3 Fusion: From Fusion06 Panel Discussion," Fusion07, 2007.

[10] E. Blasch, I. Kadar, K. Hintz, J. Biermann, C. Chong, and S. Das, "Resource Management Coordination with Level 2/3 Fusion”, IEEE AES Magazine, Mar. 2008.

[11] G. Toth, M. M. Kokar, K. Wallenius, K. B. Laskey, M. Sudit, M. Hultner, and O. Kessler, "Higher-level Information Fusion: Challenges to the Academic Community,” Panel Discussion Fusion08, 2008.

[12] S. Das, J. Llinas, G. Pavlin, D. Snyder, A. Steinberg, K. Sycara, “Agent Based Information Fusion,” Panel Discussion, Fusion07, 2007. A special issue of the Journal of Information Fusion, Elsevier Science, Vol. 11, 2010.

\section{FUSION10}

[13] P. C. G. Costa, K-C. Chang, K. Laskey, T. Levit, W. Sun, "High-Level Fusion: Steps toward a Formal Theory," Fusion10, 2010.

[14] P. Maupin, A-L Jousselme, H. Wehn, S. Mitrovic-Minic, and J. Happe, “A Situation Analysis Toolbox: Application to Coastal and Offshore Surveillance, Fusion10, 2010.

[15] J. Roy and A. B. Guyard, "Multiple hypothesis Situation Analysis Support System Prototype,” Fusion10, 2010.

[16] T. Schuck and E. Blasch, "Description of the Choquet Integral for Tactical Knowledge Representation,” Fusion10, 2010.

[17] E. Blasch, E. Dorion, P. Valin, E. Bossé, and J. Roy, "Ontology Alignment in Geographical Hard-Soft Information Fusion Systems,” Fusion10, 2010.

[18] J. Gomez-Romero, J. Garcia, M. A. Patricio, J. M. Molin, J. Llinas, S. C. Shapiro, M. Kandefer, M. Prentice, "Strategies and Techniques for Use and Exploitation of Contextual Information for High-Level Fusion Architectures," Fusion10, 2010.

[19] P. Valin, E. Bossé, A. Guitoumi, H. Wehn, and J. Happe, "Testbed for Distributed High-Level Information Fusion and Dynamic Resource Management,” Fusion10, 2010.

[20] B. Ulicny, C. J. Matheus, G. Powell, and M. M. Kokar, "Current Approaches to automated Evaluation and their Applicability to Priority Intelligence Requirement Answering," Fusion10, 2010.

[21] G. Rogova and E. Bosse, "Information quality in Information Fusion,” Fusion10, 2010.

[22] E. Blasch, P. Valin, and E. Bosse, "Measures of Effectiveness for High-Level Fusion,” Fusion10, 2010.

\section{HLIF MODELS}

[23] [JDL] US. Department of Defense, Data Fusion Sub-panel of the Joint Directors of the Laboratories, "Data Fusion Lexicon," 1991.

[24] A. N. Steinberg and C. L. Bowman, "Revisions to the JDL Data Fusion Model,” in D. L. Hall and J. Llinas (eds.) Handbook of Multisensor Fusion, CRC Press, 2001.

[25] J. Llinas, C. Bowman, G. Rogova, A. Steinberg, E. Waltz, \& F. White, "Revisiting the JDL Data Fusion Model II", Fusion2004, 2004.

[26] D. A. Lambert, "Unification of Sensor and Higher-Level Fusion,” Fusion06, 2006.

[27] M. R. Endsley, "Toward a theory of situation awareness in dynamic systems”, Human Factors 37 (1), p. 32-64, 1995.
[28] D. A. Lambert, "STDF Model Based Maritime Situation Assessments” Fusion07, 2007.

[29] Blasch, E and S. Plano, "JDL Level 5 fusion model: user refinement issues and applications in group tracking," SPIE Vol 4729, Aerosense, 2002, pp. 270 - 279.

[30] D. A. Lambert, “A Blueprint for Higher Level Fusion Systems" Journal of Information Fusion, Vol. 9 (1), p. 624, 2009.

\section{INFORMATION FUSION CHALLENGES}

[31] D. L. Hall and J. Llinas, "Introduction to Multisensor Data Fusion”, Proc., of IEEE, Vol. 85, No. 1, pp. 6 - 23, Jan 1997.

[32] D. A. Lambert, "Grand Challenges of Information Fusion," Fusion03, 2003.

[33] E Blasch, "Situation Impact and User Refinement”, 5096, SPIE 03, April 2003.

HLIF Paper Survey

[34] D. A. Lambert. "Situations for situation awareness," In. Proc. of Fusion 2001, 2001.

[35] J. Roy, "From Data Fusion to Situation Analysis," Fusion01, 2001.

[36] J Roy, S. Paradis and M. Allouche, "Threat evaluation for impact assessment in situation analysis systems," Vol. 4729, SPIE 2002.

[37] E. K. Jones, N. Denis, and D. Hunter, "Hypothesis Management for Information Fusion”, Fusion02. 2002.

[38] K. Sycara and M. Lewis, "Form Data to Actionable Knowledge and Decision”, Fusion02. 2002.

[39] K. Sycara, M. Paolucci, and M. Lewis, "Information Discovery and Fusion: Semantics on the Battlefield", Fusion03, 2003.

[40] A-L Jousselme, P Maupin, E. Bossé, "Formalization of Uncertainty in Situation Analysis,” DSTO Conf, 2003.

[41] J. Schubert, "Evidential Force Aggregation,” Fusion03, 2003.

[42] J. Hill and K. C. Chang, "Improved Representations of Sensor Exploitation in Automatic Sensor Management," Fusion03, 2003.

[43] K. Lee and J. Llinas, "Hybrid Model for Intent Estimation," Fusion03, 2003.

[44] E. P. Blasch, "Ontological Issues in Higher Levels of Information Fusion: User Refinement of the Fusion Process”, Fusion 03, July 2003.

[45] A-C. Boury-Brisset, "Ontology-based Approach for Information Fusion,” Fusion03, 2003.

[46] H. S. Carvalho, W. B. Heinzelman, A. L. Murphy, and C. J. N. Coelho, "A General Data Fusion Architecture," Fusion03, 2003.

[47] Y. Kim and T. Kesavadas, "Automated Dynamic Symbology for Visualization of High-Level Fusion," Fusion04, 2004.

[48] J. Salerno, M. Hinman, and D. Boulware, "Building a Framework for Situational Awareness,” Fusion 04, 2004.

[49] P. D. Scott and G. L. Rogova, "Crisis Management in a Data Fusion Synthetic Task Environment,” Fusion04, 2004.

[50] J. Schubert and P. Svensson, "Methodology for guaranteed and robust high level fusion performance: a literature study”, FOI-D-0216-SE, May 2005.

[51] C. Nowak and D. A. Lambert, "The Semantic Challenge for Situation Assessments,” Fusion05, 2005. 
[52] M. M. Kokar, K. Baclawski, and H. Gao, "Category Theory-based Synthesis of a Higher-Level Fusion Algorithm: An Example”, Fusion05, 2005.

[53] C. Matheus M. Kokar, K. Baclawski, J. A. Letkowski, C. Call, et al, "SAWA: an assistant for higher-level fusion and situation awareness Proc. SPIE, 2005.

[54] J. Salerno, E. Blasch, M. Hinman, and D. Boulware, "Evaluating Algorithmic Techniques in Supporting Situation Awareness,” SPIE 2005, April 2005.

[55] A. Nedich, M. Schneider, and B. Washburn, "Farsighted Sensor Management Strategies for Move/Stop Tracking," Fusion05, 2005.

[56] C.-Y. Chong and S. Mori, "Distributed Fusion and Communication Management for target Identification," Fusion05, 2005.

[57] E. Blasch, "DFIG Level 5 (User Refinement) issues supporting Situational Assessment Reasoning,” Fusion05, 2005.

[58] D. A. Lambert, "Formal Theories for Semantic Fusion," Fusion06, 2006.

[59] D. A. Lambert, "Unification of Sensor and High-Level Fusion," Fusion06, 2006.

[60] J. Holsopple, S. J. Yang, and M. Sudit, "TANDI: Threat Assessment of Network Data and Information," SPIE06, 2006.

[61] E. Blasch, "Sensor, User, Mission (SUM) Resource Management and Their interaction with Level 2/3 Fusion," Fusion06, 2006.

[62] P. Svensson, "On reliability and trustworthiness of highlevel fusion-based decision support systems: basic concepts and possible formal methodologies," Fusion 06, 2006.

[63] C. Laudy, J-G. Ganascia, and C. Sedogbo, "High-Level Fusion based on Conceptual Graphs,” Fusion07, 2007.

[64] D. A. Lambert, "Tradeoffs in the Design of Higher-level Fusion Systems," Fusion07, 2007.

[65] A. Karlsson, R. Johansson, and S. F. Andler, "An Empirical Comparison of Bayesian and Credal Networks for Dependable High-Level Information Fusion,” Fusion08, 2008.

[66] M. Witkowski, G. Whitee, P. Louvieris, G. Gorbil, E. Gelenbe, and L. Dodd, "High-Level Information Fusion and Mission Planning in Highly Anisotropic Threat Spaces," Fusion08, 2008.

[67] G. Chen, D. Shen, C. Kwan, J. Cruz, M. Kruger, E. Blasch, "Game Theoretic Approach to Threat Prediction and Situation Awareness,” Advances in Journal for Information Fusion, July 2008.

[68] A. Stotz, R. Nagi, and M. Sudit, "Incremental Graph Matching for Situation Awareness," Fusion09, 2009.

[69] A. Dahlbom and L. Niklasson, "Evolving Petri Net Situation Templates for Situation Recognition," Skövde Workshop on Information Fusion Topics (SWIFT), 2009.

[70] P. C. G. Costa, K-C. Chang, K. B. Laskey, and R. N. Carvalho, "A Multi-Disciplinary Approach to High Level Fusion in Predictive Situational Awareness,” Fusion09, 2009.

[71] P. Krauthausen and U. D. Hanebeck, "Intention Recognition for Partial-Order Plans Using Dynamic Bayesian Networks, Fusion09, 2009.

[72] D. Vincen, D. Stampouli, and G. Powell, "Foundations for System Implementation for a Centralized Intelligence Fusion Framework for Emergency Services,” Fusion09, 2009.
[73] A. N. Steinberg, "Context-Sensitive Data Fusion using Structural Equation Modeling,” Fusion09, 2009.

[74] J. Gomez-Romero, M. A. Patricio, J. Garcia, and J. M. Molina, "Ontological representation of context knowledge for visual data fusion,” Fusion09, 2009.

[75] E. Blasch, P. Valin, E. Bossé, M. Nilsson, J. Van Laere, and E. Shahbazian, "Implication of Culture: User Roles in Information Fusion for Enhanced Situational Understanding," Fusion09, 2009.

[76] C. Laudy and B. Goujon, "Soft Data Analysis within a Decision Support System,” Fusion09, 2009.

[77] P. Hilletofth, S. Ujvari, and R. Johansson, "Agent-Based Simulation Fusion for Improved Decision Making for Service Operations,” Fusion09, 2009.

[78] R. E. T. Jones, E. S. Connors, and M. Endsley, "Incorporating the Human Analyst into the Data Fusion Process by Modeling Situation Awareness Using Fuzzy Cognitive Maps,” Fusion09, 2009.

[79] T. Damarla, D. J. Thornley, D. F. Gillies, and E. Gentle, "Toward efficient quality of information estimation in simultaneous acoustic tracking and classification of multiple targets," Fusion09, 2009.

[80] M. Nilsson and J. Van Laere, "Experts' Views on User Activities in Information Fusion Systems developments Processes,” Skövde Workshop on Information Fusion Topics (SWIFT), 2009.

[81] M. Kam, X. Zhu, and P. Kalata, "Sensor Fusion for Mobile Robot Navigation,” Proc. IEEE, Vol. 85, No. 1, Jan 1997. 